\title{
Theoretical Perspectives of the Effect of Immunotherapy on the Quality of Life of Cancer Patients
}

\author{
By Swapna Johncy
}

\begin{abstract}
Immunotherapy is a type of treatment that improves an individual's ability to detect and attack cancerous tissues. The main objective of adopting immunotherapy in cancer treatment is to improve the patient's quality of life $(Q o L)$. To address the problems faced by cancer patients post immunotherapy, it is critical to conduct an extensive review to understand the factors impacting the QoL of immunotherapy patients. Quality of life is a concept very relevant to the discipline of nursing. With instrument development and population description, oncology nurses have improved the quality of life in cancer patients. The theoretical proposition of behavior change may be the best fit for understanding the difference in cancer patients' quality of life after immunotherapy, but it has limited scope of application considering its deficit in theoretical components. In cooperation with doctors and other health professionals, nurses need to develop a conceptual framework of QoL specific to immunotherapy to measure patient-reported outcomes in this cohort of patients.
\end{abstract}

Keywords: immunotherapy, cancer, quality of life, cancer theory, cancer patient

\section{Introduction}

Immunotherapy is a type of treatment that improves an individual's ability to detect and attack cancerous tissues. The advancement of immunotherapy is fundamental to enable cancer patients to obtain the internal capability to fight exacerbation attributed to cancer (Riley et al. 2019). Most cancerous cells are likely to be prevented by the immune system's ability to avoid such cells' multiplication before they become chronic. This aspect is made possible by the immune-surveillance activity that patrols the body to detect precancerous conditions. The purpose of immune surveillance is to remove potentially cancerous cells before they build up to our critical mass and develop into cancer. The main objective of adopting immunotherapy in cancer treatment is to improve the patient's quality of life.

The World Health Organisation (WHO) defines the quality of life as an individual's perception of life, objectives, values, standards, and interest in the domain of culture (Nayak et al. 2017). Healthcare stakeholders are increasingly using quality of life as a primary outcome measure in evaluating the efficiency and effectiveness of treatment. The fundamental importance in oncology is assessing the quality of life in cancer patients, considering that this metric is vital in determining the oncology unit's Endpoint of Treatment. This paper aims to examine the theoretical perspectives and understand the effect of immunotherapy on the quality of life for cancer patients.

*Advanced Practice Registered Nurse, U T MD Anderson Cancer Center and PhD Student, Texas Woman's University, USA. 


\section{Problem Identification}

Some of the significant problems facing long-term cancer survivors are emotional and social support, spiritual orientation to life, health habit, body image concern, and significant philosophical perspective (Mayer et al. 2017). However, these are the significant determinants of quality of life among cancer patients. Different studies have shown that there are other concerns among cancer survivors, including psychosocial and physical issues such as lymphedema and pain and the adverse effect of chemotherapy on the overall quality of life. The checkpoint inhibition is related to Immune-related adverse events (irAEs). Typically, these are toxicities caused by nonspecific activation of the immune system, some of which can be dangerous and life-threatening and, therefore, substantially affect patients' quality of life.

According to Miller et al. (2019), patients are assuming increased responsibility in medical treatment due to the adherence to autonomy by healthcare professionals. The improvement in cancer patients' overall outcomes is determined by their expectations and knowledge concerning the treatment options. This implies that negative expectations increase the risk of treatment side effects or nonadherence. Therefore, it is fundamental to optimism on the individual expectation to enable constipation to attain an improved quality of life in the long run. The development of new technology in oncology has necessitated stakeholders to shift towards the new approaches that are most likely to improve patients' quality of life. Clinical observations have shown that many patients are skeptical about the traditional cancer treatment method, including CT, due to the negative perceptions of adverse side effects such as mucosal membrane damage, hair loss, and nausea. Also, some of the patients believe that chemotherapy is poisonous and will lead to an insignificant effect on the quality of life (Arndt et al. 2017).

To address the problems faced by cancer patients post immunotherapy, it is critical to conduct an extensive review to understand the factors impacting the QoL of immunotherapy patients. This undertaking is particularly essential in advancing the knowledge concerning the quality of life and developing a theoretical framework for assessing the QoL of immunotherapy patients.

\section{Literature Search}

The literature search was performed using credible databases such as MEDLINE with full texts, PubMed, and Google Scholar. The inclusion criteria incorporated those articles that were published in less than ten years. Besides, the study included those articles that were only written in English. Those articles that were written in other languages were excluded from the study. The review also included articles that focused on quality of life after immunotherapy. Reports that focused on different approaches to cancer were excluded from the study. The search strategy yielded 49 articles. After filtering the articles for duplications and applying inclusion and exclusion criteria, the study attained 12 papers retained for the current review. 


\section{Data Evaluation}

Evaluation of primary sources is a technical activity and requires following strict guidelines towards this aspect. The scope of data evaluation incorporates assessing the overall quality of evidence presented related to the study parameters. However, evaluating primary sources with multiple data is a complex process due to differences in methodological approaches. Different researchers have explored the concept of the relationship between immunotherapy and quality of life in cancer patients. These articles were employed in the current study to understand the topic of interest and the attached theoretical underpinnings.

One of the critical factors in evaluating primary research is the researchers' quality and level of evidence. This is due to the possession of different author's propositions concerning the topic of immunotherapy. For example, Mamoor et al. (2020) adopted cross-sectional surveys and chat reviews of patients with advanced melanoma. The inclusion criteria included patient that had lived more than one year after initiation of treatment. Data were collected by administering a survey between February and August 2018 among the sampled participants. In total, 90 patients were included in the study. Therefore, data evaluation in this context is an impetus parameter to understand the author's perspectives concerning the study topic.

\section{Data Analysis}

Every article included in the study was analyzed to increase the understanding of the theoretical propositions concerning the quality of life in cancer patients after immunotherapy. A preliminary analysis indicated that immunotherapy was essential to improve the participants' quality of life, although patients reported moderate anxiety and depression, and pain. The authors concluded that checkpoint inhibitors contributed to significant improvement in life quality with minimal effects such as fatigue. In this respect, immunotherapy can be fundamental in optimizing clinical outcomes for cancer patients and, therefore, improve the overall quality of life in the long run.

Faury and Foucaud (2020) assessed health-related quality of life in cancer patients treated with immune checkpoint inhibitors. According to the authors, the use of immune checkpoint inhibitors has been shown to advance tremendous cancer treatment promise. One of the invaluable parameters for consideration in using immunotherapy is the type of treatment that is well tolerated in terms of health-related quality of life compared to traditional cancer therapy. The authors conducted a systematic review to evaluate the impact of immunotherapy on cancer patients' quality of life. The search included analyzing relevant databases with credible and peer-reviewed information related to the application of immunotherapy in treating cancer. The authors indicated that checkpoint inhibition could be invaluable in enabling cancer patients to manage their health outcomes by giving them autonomy to fight cancerous tissues. Accordingly, healthcare providers' responsibility is to adopt innovative approaches that will identify potential 
cancerous tissues, such as checkpoint inhibition. Application allowed the researchers to attain objectivity concerning the study topic and compare different researchers' viewpoints related to interest.

\section{Theoretical Framework Discussion}

The diverse elements that significantly affect the "QOL" of individuals and social groups are each subject, at least in principle, to some form of measurement, but no satisfactory method has yet been devised whereby these different measurements could be reduced to a single metric (Johnston, 1988). Bender and Lockey (2016) cited behavioral change theory as a prime framework to facilitate an individual orientation to healthy behavior after immunotherapy. The authors propose that the patients were most likely to positively change their quality of life after immunotherapy after adopting a healthy behavior. This framework may be essential to enhance an individual's change towards a high-quality life after immunotherapy instances. While this theoretical proposition may be the best fit for understanding the difference in cancer patients' quality of life after immunotherapy, it is limited in the scope of application considering its deficit in theoretical components. Essentially, a theory should incorporate different parameters such as definitions, assumptions, or a conceptual model to increase individual understanding of study parameters. The behavioral change model is based on an individual's behavior to influence their overall quality of life and, therefore, limited definitions and concepts.

Agur et al. (2016) applied the complex systems theory to understand cancer patients' perception of immunotherapy. The authors noted that it is critical to adopt a diverse theoretical approach to attain a more comprehensive perspective concerning cancer treatment complexities. The use of a complex systems theory allows health providers to increase care coordination among cancer patients. The authors collected patient's data as they transitioned through the complex healthcare system. One of the critical outcomes was that using complex systems bridged the communication between patient care, improving their overall experience in this perspective.

The most popular conceptual models used in cancer patients are Ferrell and Colleagues City of Hope Model and Ferrans Power QoL Model.

First of all, Ferrell and colleagues used the Padilla's of Grant's conceptual basis as a theoretical basis. Morris, et al. (1986) provided a useful definition of QOL as, "The prevention and alleviation of physical and mental suffering, Fen-el/ et al. 11 and the presence of a supportive network of informal relationships" (p. 47). This definition includes dimensions of physical and psychological well-being as well as social concerns, each of which are accepted domains of QOL (Morris et al. 1986). In 1989, Ferrel et al. used the Padilla and Grant model as a conceptual framework to develop and test the quality of life instruments.

A conceptual model emerged from these two studies and was referred to as the City of Hope Model. This model illustrated the influence of pain on the dimension of quality of life. The model supports that quality of life has four 
dimensions: physical well-being and symptoms, psychological well-being, social well-being, and spiritual well-being. In this model, it was demonstrated that pain is an experience that influences all dimensions of QoL (Chopra and Kamal 2012). Later it was demonstrated that fatigue is a variable that influences all four dimensions of QoL (Ferrans, 2004).

Ferrans and Powers (1985), in his conceptual model, described four significant domains of QoL: health and functioning, socioeconomic psychological, spiritual, and family (Moons et al. 2006). The four domains include 35 aspects of life conveying the multi-dimensionality of the concept. Ferran's framework was based on a literature review and statistical analysis using data from patients undergoing hemodialysis. The initial QLI was modified and tested with a population of clients with cancer. Ferrans (2004), in his paper, supported that "the model was developed based on the adoption of an individualistic ideology, which recognizes that quality of life depends on the unique experience of life for each person. Individuals are the only proper judge of their quality of life because people differ in what they value. Consistent with this ideology, quality of life was defined in terms of satisfaction with the aspects of life that are important to the individual" (Moons et al. 2006).

\section{Patient's Expectations}

Patient's expectations concerning immunotherapy contribute significantly to an overall improvement in quality of life. According to a study conducted by Ihrig et al. (2020), cancer patients have higher expectations for immunotherapy compared to traditional chemotherapy for cancer. This study aimed to analyze the knowledge and expectation of patients with advanced-stage cancer concerning immunotherapy. The authors indicated that treatment expectations are complex and can be traced back to several information sources. In this respect, the study adopted qualitative and quantitative research approaches to obtain an in-depth understanding of the research purpose. To get a clear distinction between immunotherapy and traditional chemotherapy, the study included 55 cancer patients undergoing chemotherapy, 53 patients on immune checkpoint inhibitors, and 53 non-cancer patients. Patients' expectations concerning immunotherapy and chemotherapy were compared to obtain the study metrics' orientation. Besides, qualitative data were derived from semi-structured interviews.

The results indicated that the participants perceived immunotherapy positively regardless of whether they had heard or received such interventions compared to chemotherapy. One of the significant outcomes out of the study was their lack of proper knowledge and information. The participants also listed immunotherapy as being more promising with minimal side effects compared to those of chemotherapy. However, some participants experienced adverse effects that were well tolerated and mostly mild or easily treated.

The evidence presented by O'Reilly et al. (2019) is critical in understanding the importance of immunology in improving cancer patients' quality of life. The authors' proposed to assess the health-related quality of life and toxicity in patients with melanoma treated with immune checkpoints inhibitors. According to the 
authors, the use of immune checkpoint inhibitors has culminated in a subgroup of patients with metastatic melanoma. The study's interest was to assess the immunerelated adverse effects and the exposure to immunosuppressive agents on the patient's quality of life. The study hypothesized that immunotherapy techniques could be critical in improving cancer patients' quality of life.

The study used questionnaires to collect data from sampled patients in the UK. A sample of 36 questionnaires was used to assess the health-related quality of life among the sampled participants. The authors evaluated the patient's electronic health data, including the relevant demographics, to determine the effects of the immunotherapy interventions on patients' quality of life. The study has high reliability and validity, considering that an adequate sample of participants aged 18-39 was employed in the study. The age-based methodology in data collection could be critical to determine individuals' subjective responses based on age and demographic variables.

The participants' results were analyzed using a comparative approach whereby an independent t-test was used to compare group tests. The study used the ANOVA approach in analyzing data demographic data based on traditional sources and evaluated by applying a one-sample t-test. The findings indicated that the immunotherapy technique for cancer treatment reduced toxicity among the participants compared to conventional chemotherapy. The outcome also implicated immunotherapy techniques for a higher quality of life regarding social, physical, and psychosocial functioning at the general health than the normative population. However, the findings illustrate a significant reduction in quality of life among the patients who had previous exposure to ipilimumab than those who did not have to use the drug. Therefore, the results concluded that metastatic melanoma survivors had a higher potential to increase the quality of life and reduced impairment attributed to immunotherapy. This outcome was significant, especially for patients with other comorbidities compared to healthy participants.

\section{Health-Related Quality of Life}

Evaluating health-related quality of life among the patients is an essential parameter in assessing the treatment efficacy. This was the focus by Park et al. (2020) in evaluating patient preferences after receiving immunotherapy for stage 4 Non-small-cell lung cancer. Also, the study is targeted at assessing the views of the family and friends concerning the cancer treatment modality and the determinant of the quality of life of long-term survivors. According to the study, it is fundamental for stakeholders in the oncology sector to adopt interventions that will lead to positive outcomes among cancer patients. In this respect, the application of immunotherapy can be critical to increase the patient's quality of life and overall recovery post-medication. The authors adopted ethnographic research by applying 24 patients who had responded to immune-oncology treatment. This study could attain high internal validity due to the inclusion of samples from three countries, including the US, UK, and Denmark. 
Jackson et al. (2015) conducted a similar study to evaluate the association between quality of life in cancer patients and the use of immunotherapy techniques for oral health. According to the authors, one of the success measures among cancer patients is improved quality of life. The specific objectives were to assess immunotherapy for individuals with oral cancer, a critical parameter in determining such interventions' efficacy. These approaches were similar to that of Park et al. (2020) in assessing the interest parameters. The adoption of the researcher's observation approach was critical to enable the researchers to adopt objectivity concerning the study metrics. The findings indicated that using immunotherapy techniques in addressing cancer was essential to increase patients' survival rates. The results also posited that those patients who attained immunotherapy had a higher quality of life concerning reduced pain and increased recovery speed than their initial prognosis. Besides, these participants were able to attain normalcy semblance despite their identification as individuals with terminal illnesses. This aspect impacted their decision-making, quality of life, patient support experience, and ability to seek health promotion behaviors. Generally, the authors recommend using immunotherapy approaches to increase the quality of life among cancer patients.

\section{Limitations}

The review earned limitations in the lack of a straightforward theoretical approach to understanding the quality of life after immunotherapy which is evident by the inclusion of 12 articles that satisfactorily met the required threshold. The review's interest was to include more than 20 articles to increase the reliability and validity of data attained. However, there were limitations in the scope and length of such undertakings, considering the time constraints.

\section{Conclusion}

Quality of life is a concept very relevant to the discipline of nursing. With instrument development and population description, oncology nurses have improved the quality of life in cancer patients. The theoretical proposition of behavior change may be the best fit for understanding the difference in cancer patients' quality of life after immunotherapy, but it has limited scope of application considering its deficit in theoretical components.

Complex systems theory concepts bridged the communication between patient and care providers, improving their overall treatment experience. Ferrans, in his conceptual model, described four significant domains of QoL: health and functioning, socioeconomic psychological/spiritual, and family. The City of Hope model supports that quality of life has four dimensions: physical well-being and symptoms, psychological well-being, social well-being, and spiritual well-being. This model demonstrated that pain and fatigue are the key variables that influence all dimensions of QoL. 
In cooperation with doctors and other health professionals, nurses need to develop a conceptual framework of QoL specific to immunotherapy to measure patient-reported outcomes in this cohort of patients. Oncology nurses will continue to evaluate the impact of cancer and cancer treatment on quality of life and do researches for strategies to decrease adverse physical, psychological, social, and spiritual effects on the lives of cancer patients.

\section{References}

Agur Z, Halevi-Tobias K, Kogan Y, Shlagman O (2016) Employing dynamical computational models for personalizing cancer immunotherapy. Expert Opinion on Biological Therapy 16(11): 1373-1385.

Arndt V, Koch-Gallenkamp L, Jansen L, Bertram H, Eberle A, Holleczek B, et al. (2017) Quality of life in long-term and very long-term cancer survivors versus population controls in Germany. Acta Oncologica 56(2): 190-197.

Bender BG, Lockey RF (2016) Solving the problem of nonadherence to immunotherapy. Immunology and Allergy Clinics 36(1): 205-213.

Chopra I, Kamal KM (2012) A systematic review of quality of life instruments in longterm breast cancer survivors. Health and Quality of Life Outcomes; Health Qual Life Outcomes 10(1): 14.

Faury S, Foucaud J (2020) Health-related quality of life in cancer patients treated with immune checkpoint inhibitors: a systematic review on reporting of methods in randomized controlled trials. PloS One 15(1): e0227344.

Ferrans CE (2004) Definitions and conceptual models of quality of life. Cambridge University Press.

Ferrans C, Powers M (1985) Quality of life index: development and psychometric properties. Advances in Nursing Science 8(1): 15-24.

Ferrell BR, Wisdom C, Wenzl C (1989) Quality of life as an outcome variable in the management of cancer pain. Cancer 63(11): 2321-2327.

Ihrig A, Richter J, Grüllich C, Apostolidis L, Horak P, Villalobos M, et al. (2020) Patient expectations are better for immunotherapy than traditional chemotherapy for cancer. Journal of Cancer Research and Clinical Oncology 146(12): 3189-3198.

Jackson LK, Johnson DB, Sosman JA, Murphy BA, Epstein JB (2015) Oral health in oncology: impact of immunotherapy. Supportive Care in Cancer 23(1): 1-3.

Johnston DF (1988) Toward a comprehensive 'quality-of-life' index. Social Indicators Research 20(5): 473-496.

Mamoor M, Postow MA, Lavery JA, Baxi SS, Khan N, Mao JJ, et al. (2020) Quality of life in long-term survivors of advanced melanoma treated with checkpoint inhibitors. Journal for Immunotherapy of Cancer 8(1): e000260.

Mayer DK, Nasso SF, Earp JA (2017) Defining cancer survivors, their needs, and perspectives on survivorship health care in the USA. The Lancet Oncology 18(1): e11-e18.

Miller KD, Nogueira L, Mariotto AB, Rowland JH, Yabroff KR, Alfano CM, et al. (2019) Cancer treatment and survivorship statistics, 2019. CA: A Cancer Journal for Clinicians 69(5): 363-385.

Moons P, Budts W, De Geest S (2006) Critique on the conceptualisation of quality of life: a review and evaluation of different conceptual approaches. International Journal of Nursing Studies 43(7): 891-901. 
Morris J, Suissa S, Sherwood S, Wright S, Greer D (1986) Last days: a study of the quality of life of terminally ill cancer patients. Journal of Chronic Diseases 39(1): 47-62.

Nayak MG, George A, Vidyasagar MS, Mathew S, Nayak S, Nayak BS, et al. (2017) Quality of life among cancer patients. Indian Journal of Palliative Care 23(4): 445450.

O'Reilly A, Hughes P, Mann J, Lai Z, Teh JJ, Mclean E, et al. (2019) An immunotherapy survivor population: health-related quality of life and toxicity in patients with metastatic melanoma treated with immune checkpoint inhibitors. Supportive Care in Cancer 28(2): 561-570.

Park R, Shaw JW, Korn A, McAuliffe J (2020) The value of immunotherapy for survivors of stage IV non-small cell lung cancer: patient perspectives on quality of life. Journal of Cancer Survivorship 14(3): 363-376.

Riley RS, June CH, Langer R, Mitchell MJ (2019) Delivery technologies for cancer immunotherapy. Nature Reviews Drug Discovery 18(3): 175-196. 
